\section{Issues with anti-Gr1 antibody-mediated myeloid-derived suppressor cell depletion}

We read with great interest the article 'Myeloid-derived suppressor cells have a proinflammatory role in the pathogenesis of autoimmune arthritis' by Chunqing Guo et al. ${ }^{1}$ In this paper, the authors used anti-Gr1 antibody to deplete myeloid-derived suppressor cells (MDSCs) in arthritic mice and they found that it reduced disease severity and Th17 response. However, they did not report the efficiency of MDSC depletion.

Anti-Gr1 antibody (RB6-8C5) was widely used and considered to be effective in eliminating MDSC. Srivastava et $a l^{2}$ found that anti-Gr1 antibody led to a reduction in $\mathrm{Gr}^{+}$cells in tumour, blood, spleen and bone marrow (BM). Vincent Hurez used anti-Gr1 monoclonal antibody, which reduced MDSCs by $50 \%-75 \%$ in the spleen of tumour bearing (TB) mice, without reporting the results in $\mathrm{BM}$ and tumour. ${ }^{3}$ Zhang et $a l^{4}$ found that anti-Gr1 antibody reduced MDSC by one-third in tumour. Thomas Condamine et al determined that anti-Gr1 antibody eliminated about 95\% of MDSCs in spleen and blood of TB mice; however, it raised the immature myeloid cell (IMC) levels in the BM. ${ }^{5} \mathrm{Ma}$ et $a l^{6}$ and Kumar et $a l^{7}$ believed that anti-Gr1 antibody could not eliminate Ly6 $\mathrm{C}^{\text {high }}$ MDSCs. Besides, Ma et $a l^{6}$ first indentified that anti-Gr1 antibody failed to reduce MDSCs in the liver. The liver might generate a more favourable environment for MDSCs. ${ }^{5}$ The present study did not present the efficacy of depletion at disease sites, spleen and BM.

The efficacy of anti-Gr1 antibody was controversial. In the field of cancer, Srivastava et al, Zhang et al and many other researchers found that depletion of MDSCs by anti-Gr1 antibody led to the inhibition of tumour volume and tumour weight. ${ }^{2} 4$ The results of Hurez et $a l^{3}$ were different. Anti-Gr1-mediated depletion of MDSCs resulted in significantly slower tumour growth in the aged but not the young B16-bearing mice. The study by Kumar et $a l^{7}$ did not find the anti-tumour efficacy of anti-Gr1 antibody. This inconsistence might influence other modes, such as arthritic mice in the present study.

In summary, anti-Gr1 antibody (RB6-8C5) is widely used as an efficient agent for eliminating MDSCs in mice; however, its efficacy on each subtype of MDSCs, polymorphonuclear neutrophil MDSC (PMN-MDSC) and monocyte MDSC (M-MDSC) is still controversial. Meanwhile, there are more debates ongoing about its efficacy in disease control. Using novel methods to deplete MDSCs shall be an acceptable choice. ${ }^{8}$

\section{Yan-Fang Xing, ${ }^{1}$ Yu-Qi Zhou, ${ }^{2}$ Guo-Wei Ma, ${ }^{3}$ Ding-Yun Feng, ${ }^{2}$ Xiu-Rong Cai, ${ }^{4}$ Xing $\mathrm{Li}^{4}$}

1 Department of Nephrology, The Third Affiliated Hospital of Guangzhou Medical University, Guangzhou, People's Republic of China

${ }^{2}$ Department of Respiration, The Third Affiliated Hospital of Sun Yat-sen University, Guangzhou, People's Republic of China
${ }^{3}$ Sun Yat-sen University Cancer Center, State Key Laboratory of Oncology in South China, Guangzhou, People's Republic of China

${ }^{4}$ Department of Medical Oncology, The Third Affiliated Hospital of Sun Yat-sen University, Guangzhou, People's Republic of China

Correspondence to Dr Xing Li, Department of Medical Oncology, The Third Affiliated Hospital of Sun Yat-sen University, 600 Tianhe Road, Guangzhou 510630, China; lixing9@mail.sysu.edu.cn

Contributors All authors have read and approved the final manuscript.

Funding This study was supported by the Natural Science Foundation of Guangdong (No. 2014A030313146 and 2016A030313302).

Competing interests None declared.

\section{Patient consent Obtained.}

Provenance and peer review Not commissioned; internally peer reviewed.

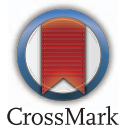

To cite Xing Y-F, Zhou Y-Q, Ma G-W, et al. Ann Rheum Dis 2016;75:e49.

Received 25 April 2016

Revised 4 May 2016

Accepted 5 May 2016

Published Online First 25 May 2016

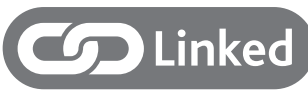

http://dx.doi.org/10.1136/annrheumdis-2016-209848

Ann Rheum Dis 2016;75:e49. doi:10.1136/annrheumdis-2016-209786

\section{REFERENCES}

1 Guo C, Hu F, Yi H, et al. Myeloid-derived suppressor cells have a proinflammatory role in the pathogenesis of autoimmune arthritis. Ann Rheum Dis 2016;75:278-85.

2 Srivastava MK, Zhu L, Harris-White M, et al. Myeloid suppressor cell depletion augments antitumor activity in lung cancer. PLOS ONE 2012;7: e40677.

3 Hurez V, Daniel BJ, Sun L, et al. Mitigating age-related immune dysfunction heightens the efficacy of tumor immunotherapy in aged mice. Cancer Res 2012;72:2089-99.

4 Zhang $Y$, Liu Q, Zhang $M$, et al. Fas signal promotes lung cancer growth by recruiting myeloid-derived suppressor cells via cancer cell-derived PGE2. J Immunol 2009;182:3801-8.

5 Condamine T, Kumar V, Ramachandran IR, et al. ER stress regulates myeloid-derived suppressor cell fate through TRAIL-R-mediated apoptosis. J Clin Invest 2014; 124:2626-39.

6 Ma C, Kapanadze T, Gamrekelashvili J, et al. Anti-Gr-1 antibody depletion fails to eliminate hepatic myeloid-derived suppressor cells in tumor-bearing mice. J Leukoc Biol 2012;92:1199-206.

7 Kumar V, Cheng P, Condamine T, et al. CD45 phosphatase inhibits STAT3 transcription factor activity in myeloid cells and promotes tumor-associated macrophage differentiation. Immunity 2016:44:303-15.

8 Qin H, Lerman B, Sakamaki I, et al. Generation of a new therapeutic peptide that depletes myeloid-derived suppressor cells in tumor-bearing mice. Nat Med 2014;20:676-81. 